\title{
A multi-media approach to health education*
}

\author{
R C FRIEDERICKS \\ Development Communication Productions Kathmandu, Nepal
}

Many health and development agencies around the world concentrate on providing services, training personnel, supplying materials, etc., and overlook the part communication plays in their activities. Many books and manuals available on development issues do not address communications. Yet it is so much a part of their activities that it is taken for granted. But a close look at the communication and the information components of a programme can be beneficial for the success of the whole project.

Every kind of health or development activity is information related or dependent in some way.' These information components can and should be identified. Successful programmes are the ones that are involved with and aware of the people they serve. They are set up to pass on materials and technology that are appropriate to the situation and are oriented to giving away information.

Most of the information components involved in development or health education communication can be summarized in 'prime or key messages' which are relatively easy to transfer. ${ }^{2}$ It is sometimes assumed that communication brings about change in peoples lives. Health education by itself can not make a difference because along with health education there must be an infrastructure of clinics, medical personnel and medicines. Only a better mix of information and human, physical and material sources can bring about substantial changes. ${ }^{3}$

People must have access to material resources as well as to information. Communication must lead to awareness and to feasible action. Using the radio to inform all pregnant women to go to the nearest hospital at the first sign of labour will not be helpful to a woman living 10 days walk from a hospital. To use a commercial example, it is frustrating to many young people living in the remote hills of Nepal to continually hear messages on the radio encouraging them to enjoy some commodity which is not available in their district.

Therefore information must be appropriate to the availability of services and materials where the information is distributed. These considerations help communicators define the information that is essential and produce messages that are most helpful to the people.

\footnotetext{
* Paper presented at a Health Education Seminar sponsored by the International Nepal Fellowship, Pokhara, Nepal on 23 March 1990
} 


\section{Variety of media}

Media can be categorized into basic and extended media. ${ }^{4}$ Basic media are those which communicate ideas in the basic environment; person to person, person to group, group discussions, classroom lectures, or even large public meetings where public address systems are used. Extended media, on the other hand, are those media which extend time and space; books, other printed materials, films, audio cassettes, television, and even the telephone. In the basic media situation various extended media can be incorporated such as posters, flashcards, slides, audio cassettes, and other visual aids which help focus attention or expand the impact of the message.

No one media is better than another. Each media has its function, and each one is useful for a specific purpose. Combining them often increases their usefulness. Commercial advertising has used every possible medium and has often lead the way in the field of communication. ${ }^{5}$ Many of the principles and techniques of developmental communication have been borrowed from commercial advertising.

\section{Multi-media approach}

One of the principles discovered was that in order to reach every possible target group it was necessary to use as many media as possible. The same product will be advertised in many magazines targeted for different audiences, radio programmes of many kinds, TV ads during many different kinds of programmes, and then, of course, in brochures, posters, bill boards along highways and on buses. The general public is assaulted with images and phrases about the product. Through this the public is informed, whether it wants to be or not, of the product and its qualities.

Similar approaches have been successfully used for health education. In India over the last few years, WHO and other funding agencies have given about $\$ 20$ million for the national EPI (immunization) programme. A full $10 \%$ of this, $\$ 2$ million, was spent on communication. The mass media was used, extension workers equipped with visual aids were dispatched, posters, bill boards, leaflets, were all produced and distributed. This approach was so successful that there has been a tremendous demand for immunization throughout India, almost more than the EPI programme can handle. ${ }^{6}$

In Nepal the Nun Chini Pani (oral rehydration) campaign used a similar multi-media approach. Radio, television, demonstrations by extension workers in village squares and schools were carried out throughout the country. Posters, memory cards, leaflets, and many other items, each giving the directions for making the oral rehydration solution with household items were distributed. At the end of the 3 year campaign $85 \%$ of the population of Nepal had heard the message, $57 \%$ could repeat the ingredients, and $25 \%$ had actually used it. Of those who had heard the message it was reported that $60 \%$ had heard it on the radio. ${ }^{7}$

Though radio may have been the original source of hearing about oral rehydration for $60 \%$ of the population, it is likely that their understanding of the message and ability to repeat or use the information was reinforced through other media such as the mobile teams who actually demonstrated the mixing of the ingredients, or from posters, leaflets or other materials.

In both cases mentioned above, it was determined that success was due to continuous, 
rather than sporadic, repetition of simple messages over a long period of time using all available media. The campaigns were based on solid research into local needs, beliefs, attitudes, knowledge and practices. Interpersonal channels of communication were by far the most important because of personal contact and an opportunity to actually demonstrate or answer questions. Posters, the radio and television were the most effective in creating awareness. Radio and TV messages lent authority to the teams who came to villages to talk and demonstrate and helped mould public opinion and create a receptive attitude. Personal visits and demonstrations imparted the actual techniques or encouraged the decision making process toward trial and use of the oral rehydration solution or immunization of children.

Health and development programmes need to take a careful look at how they are communicating. Many rely only on basic media. Others use extended media as an aid or in some cases in place of basic media. A poster may be put up on a clinic wall and the staff may assume that their health education obligation has been met. Are staff trained well enough in communication skills to be effective basic communicators? Are doctors, nurses, fieldworkers, and extension workers trained as effective basic communicators? Are they able to effectively use extended media?

Basic media, if used correctly, is extremely effective for person to person contact. It is effective in the clinic with a patient or in the waiting room, in the village square or in a school. Mobile teams or travelling drama groups are likewise effective with person to person contacts. The use of audio/visual materials, which are helpful in attracting large crowds, can be turned into a basic media situation by having a discussion afterwards.

Distribution of printed materials has not proven effective in Nepal. This is due to the difficulty of carrying and distributing the material and the low literacy rate in rural areas. Visual literacy, though easy to teach, is limited primarily to those who are already literate. ${ }^{8}$ This limits the effectiveness of visual aids and posters.

The Leprosy Control Programme in Nepal made significant changes in early case finding and attitude change across Nepali society. Though the problem of leprosy has not been solved, it may be safe to assume that the level of success achieved has been due to the medical infrastructure available in many parts of the country and to the continuous repetition of simple messages:

(1) leprosy can be cured ...

(2) the early signs of leprosy are ...

(3) treatment can be found at ...

These messages have been repeated by healthworkers in the field, by printed materials distributed among community leaders who are often literate, and over the radio. It has been found that during and after periods of radio broadcast of these messages, case finding significantly increased. ${ }^{9}$

\section{A strategy for the nineties?}

If each programme commits itself to serious analysis of its communication components it will be able to identify the key messages it has to offer the people it serves. Simplicity is essential. It is also essential that the message be relevant. For example, a key message for a TB programme might be 'TB can be cured if treatment is taken regularly for a year'. This 
kind of message can be repeated through various media and is relevant to the entire population, both for those who have TB and for those who are in danger of getting the disease. This message can be repeated over the radio to create awareness and add authority to the healthworker. A leaflet can provide details to those who can read and want to know more. The messages should be based on realistic objectives related to actual local conditions and possibilities.

It will be discovered that many programmes share the same essential key messages, although there may be variations due to local culture and language. A consistent approach to communicating these key messages is necessary. All health and development programmes would be better off if they could pool their efforts to 'sell' nutrition, sanitation, TB control or whatever. Sharing creative ideas will preclude waste, amateurish production efforts, and the 're-invention of the wheel'.

Identifying key messages and designing them for the various media requires a central resource. This resource would need to assume a role in co-ordinating the identification of key messages, research and design for appropriate communication strategies, and the production of materials at a national or regional level for various health and development programmes. The mandate of such a resource centre would be the effective utilization of communication as a powerful tool for health and development.

\section{References}

1 McAnany EG. Communications in the rural third world: the role of information in development. New York: Praeger, 1980, p. 5.

2 Facts for life: a communication challenge, UNICEF, WHO, and UNESCO, P\&LA, UK.

3 Op. cit., McAnany, p. 5.

${ }^{4}$ Sogaard V. Everything you need to know for a cassette ministry. Minneapolis: Bethany Fellowship, 1975, p. 26.

5 Baker S. Systematic approach to advertising creativity. New York: McGraw-Hill Company, 1978, p. 152.

6 Gill R. Conversation at UNICEF/Nepal, March 19, 1990.

7 Karki YB. Communicating the ORT message in Nepal. WIF, 1989.

${ }^{8}$ McBean G. Changing vision. UNICEF/Nepal, 1988.

9 Bedenbender/Hagen, '1985/86 Annual Report of the INF leprosy control programme', Pokhara, Nepal. 